\title{
ESTUDIOS EN COSTA RICA SOBRE LENGUA CASTELLANA: DE GAGINI A AGUERO
}

Víctor Sánchez Corrales

\begin{abstract}
This article takes a critical retrospective look at the studies carried out in Costa Rica concerning the Spanish language, during the period 1890 to 1970. According to the main interest of each author, these studies are divided into two groups: those which refer to the educated variety of Spanish as the prestige form and as a criterion for correctness, and those which attempt a characterization of the Spanish spoken in Costa Rica as opposed to other Spanish American or peninsular speech forms.
\end{abstract}

\section{INTRODUCCION}

1.1. Los trabajos escritos en Costa Rica acerca de la lengua castellana, en el período que hemos llamado de Gagini a Agüero (1890-1970), se dividen, de acuerdo con el interés primordial de cada uno de ellos, en dos grupos:

1.1.1. Estudios cuyo énfasis es la lengua castellana, habiéndose tomado una de sus variantes como objeto de análisis y enseñanza en colegios o educación superior. Se describe y estudia la variante que se promueve, cual bien deseado, como manual de consulta en casos de vacilaciones. En este dominio ubicamos las gramáticas de Gagini (1907), Napoleón Quesada (1929), Moisés Vincenzi (1939), Hernán Zamora (1941) y Jézer González (1968), para citar las más importantes. Todas estas gramáticas hacen énfasis en la oración y sus partes, lo cual impide que se estudien más profundamente otros niveles de la lengua. Brenes Mesén (1905) difiere de los anteriores, al negar la condición de arte de la gramática y postular la descripción del uso como objetivo fundamental de ella.

1.1.2. Estudios cuyo interés primordial es individualizar la lengua castellana en Costa Rica respecto de hablas americanas o peninsulares. En este cam- po tenemos que mencionar a cuatro investigadores: Gagini (1892 y particularmente 1918), Agüero (1962), Arroyo (1971) y Gaínza (1976), quienes se propusieron darnos una visión de conjunto de los principales fenómenos lingüísticos correspondientes a nuestra lengua materna. Otros investigadores han estudiado uno de los componentes de la lengua o un tópico de ellos.

\section{ESTUDIOS CON ENFASIS EN LA LENGUA CASTELLANA}

2.1. La tradición lingüística en Costa Rica, hasta el período de Arturo Agüero, se caracteriza por la influencia decisiva de la doctrina gramatical de la Academia y de las aportaciones teóricas de don Andrés Bello, más recientemente. En ambos casos la gramática es arte; la diferencia está en el escogimiento de la modalidad lingüística que se propone como norma.

Bello defiende, ante la norma castellana, el hablar correctamente conforme al buen uso de la gente educada en América:

No se crea que recomendando la conservación del castellano sea mi ánimo tachar de vicioso y espurio todo lo que es peculiar de los americanos. Hay locuciones castizas que en la Península pasan hoy por anticuadas, y que subsisten tradicionalmente en Hispanoamérica; ¿por qué proscribir- 
las? Si según la práctica general de los americanos es más analógica la conjugación de algún verbo, ¿por qué razón hemos de preferir la que caprichosamente haya prevalecido en Castilla? Si de raíces castellanas hemos formado vocablos nuevos, según los procederes ordinarios de derivación que el castellano reconoce, y de que se ha servido y se sirve continuamente para aumentar su caudal, ¿qué motivos hay para que nos avergoncemos de usarlos? Chile y Venezuela tienen tanto derecho como Aragón y Andalucía para que se toleren sus accidentales divergencias, cuando las patrocina la costumbre uniforme y auténtica de la gente educada. En ellas se peca mucho menos contra la pureza y corrección del lenguaje que en las locuciones afrancesadas, de que no dejan de estar salpicadas hoy día aun las obras más estimadas de los escritores peninsulares. (Bello 1847/1970:25)

En oposición a la doctrina de la Academia, Bello propone la categorización de los vocablos sobre la base de su función sintáctica, lo cual le permite clasificar las partes del discurso en siete categorías, tal como se sabe.

Pues bien, la teoría gramatical académica, a caballo entre la ontología y hechos funcionales, y la gramática de Bello, tal como dijimos, son dos de las fuentes esenciales para este primer tipo de estudios lingüísticos que estamos comentando.

\subsection{La obra Gramática histórica y lógica de la} lengua castellana de Brenes Mesén (1905) constituye el primer trabajo sobre la lengua castellana que ubicamos en el período "de Gagini a Agüero". En esta obra el autor se propone describir los fenómenos de la lengua castellana, cuya fundamentación se ha de buscar en la historia interna de la lengua. Insiste, como Bello, en que el uso debe erigirse en ley gramatical y toma la oración, unidad psicológico-sintáctica autónoma, como elemento fundamental del análisis. Difiere de Bello, al desechar como norma el uso de la 'gente educada', pero la descripción que hace no se ajusta realmente a ninguna habla regional, sino que es aplicable a la lengua castellana en general. El pensamiento se patentiza lingüísticamente en unidades psicológicas y sintácticas llamadas frases, las cuales se analizan en palabras (variables e invariables) como elementos constitutivos, y estas, por último, constan de sonidos:

Todos los fenómenos que operan en la lengua se reducen a esos tres grandes grupos: los sonidos, las palabras y las frases. (Brenes Mesén 1905: XV)

El autor dedica solamente un párrafo a "los sonidos castellanos en América ", para señalar el seseo, yeísmo, $s$ aspirada y su reducción a cero, re- ducción a cero de $-d$ final, lo cual demuestra que, a pesar de su insistencia en el uso americano, no se pudo desligar de la gran influencia que ejercía la norma culta peninsular.

El mérito principal de esta obra, opinamos, es una actualizada fundamentación teórico-metodológica -para ese entonces- ya que tomó como punto de partida las aportaciones filológicas, lingü ísticas y filosóficas que estaban en boga para esos tiempos. Promete un segundo volumen sobre sintaxis y el español en América que todo parece indicar nunca escribió.

2.3. Don Carlos Gagini publica Elementos de Gramática Castellana (1907), la cual conjuga criterios diacrónicos y descriptivos, para explicar los fenómenos de lo que él considera como distintos componentes de la gramática (fonología, etimología, morfología y sintaxis).

El autor opina que es necesario aprender ciertos principios idiomáticos "para hablar bien" (Introducción, p.5). De su gramática, ha trascendido la clasificación que propone para los verbos irregulares, cuya paternidad reclama ante el plagio que se le estaba haciendo. De acuerdo con su noción de gramática como arte, la obra culmina con unos ejercicios de corrección idiomática.

\subsection{Napoleón Quesada en Lecciones de Gramática} Castellana (1929) representa un hito en los estudios gramaticales en Costa Rica, al considerar, con Cuervo y Hanssen, que tales investigadores deben extenderse a formas menos distinguidas y armoniosas (sic), incluyendo el habla de las clases populares más bajas. Debe revisarse, para su rectificación, el concepto de gramática como el arte del buen uso, propio de la gente educada:

La gramática debe considerarse como un estudio de observación que tiene un campo en extremo dilatado e interesante y digno en extremo de fijar nuestra atención: la lengua popular, viviente, siempre activa, siempre en evolución. (Quesada 1929: IV)

La descripción de los hechos observados, debe hacerse a partir de un examen lógico de los fenómenos y recurriendo al estudio histórico interno de éstos. Respecto de la fundamentación lógica rigurosa del análisis, este puede llevar al gramático a condenar el uso: "en muchos casos formas, significados, giros y construcciones que no se avienen con aquélla y sólo se fundan, por lo general, en el capricho de quienes los empleen o en el afán de "conseguir novedad léxica o sintáctica para disimu- 
lar la poquedad o la vulgaridad de las ideas; $y$ esta es precisamente la tarea gramatical que puede resultar práctica y utilitaria" (Quesada 1929: VII). Por su parte, el estudio diacrónico de los hechos puede resultar más interesante, opina el autor, que su presentación sincrónica "ofrece poquísimo interés el discutir si la forma le es preferible a lo para el acusativo masculino del pronombre de tercera persona, pues ambas tienen en su abono el uso por excelentes escritores; la importancia está en la explicación histórica de cómo la forma etimológica lo se sustituyó por la analogía le" (Quesada 1929: VII).

La tradición gramatical se le impone a Quesada al condenar formas populares como ñor, ña, mano, mana e hipocorísticos como Tavio o Tavo, Miro, Hildo, etc.:

No creemos recomendable esta práctica de desfigurar los nombres de personas, práctica tan extendida en Costa $\mathbf{R i}$ ca y que parece reflejar en el lenguaje un carácter apocado que dichosamente no es nacional. (Quesada 1929: 61)

\subsection{Con la obra de Hernán Zamora E., Gramática} castellana (1941), se pretende entronizar la teoría gramatical de Andrés Bello. El estudio de la lengua debe partir de la oración, en tanto construcción bimembre, y la categorización de las palabras debe fundamentarse en marcos sintácticos, para identificar la función privativa de cada una de ellas. El definir y clasificar a partir de significados son cuestiones de la lógica, opina el autor, y entre lógica y gramática no siempre hay acuerdos:

Por consiguiente la función debe ser la base de toda clasificación y de toda definición. (Zamora 1941:4)

Este criterio teórico impone a Zamora Elizondo un principio de organización de su trabajo: la primera parte constituye la sintaxis y la segunda, la morfología; hecho que muestra la importancia de la doctrina gramatical de Bello en esta obra.

2.6. Durante la veintena siguiente no se publica ninguna gramática castellana de importancia, excepto manuales escolares que divulgan los enfoques de la Academia o de Bello.

2.7. En la década de los sesenta, para cerrar lo que hemos llamado el período de Gagini a Agüero, en este primer tipo de estudios aparece la obra Curso fundamental de gramática castellana de Jézer González (1968, ahora con 3 ediciones), la cual se ubica en la misma línea que hemos venido presentando: describir la morfología y sintaxis de la lengua castellana en su variante literaria, sin interesarse por los otros niveles estilísticos o variantes regionales de la lengua. Esta obra persigue proporcionar al lector los principios morfosintácticos, sobre la base de ejemplos "tomados todos ellos de la lengua literaria de ayer y de hoy" (González 1968: 1).

Al referirnos a este estudio, no podemos pasar inadvertida una preocupación del autor que va más allá de la descripción gramatical. A lo largo de la obra se ve un interés manifiesto porque los principios de estructuración de la lengua literaria sean aprovechados por el estudiante en la expresión escrita. El estudio de la morfosintaxis sobre la base de textos "tomados todos ellos de la lengua literaria de ayer y de hoy", redundará en beneficio de la expresión escrita, al acatarse las reglas de concordancia (nominal, verbal, etc.) y los regímenes en cada caso, cuya consecuencia es una concatenación adecuada de los constitutos de la oración, del período, del párrafo. Teóricamente, si bien el autor mismo dice que su enfoque es tradicional, se pretende dar definiciones y clasificaciones sobre la base de criterios estrictamente lingüísticos.

2.8. Las obras que hemos comentado anteriormente, a excepción de Brenes Mesén (1905), nos muestran una preocupación común y constante: la gramática está al servicio del hablar conforme a la gente culta o del escribir de acuerdo con los patrones de la lengua literaria. Esta tradición lingüística es la que ha sustentado la enseñanza de la gramática en escuelas, colegios y educación superior costarricense hasta años recientes.

\section{ESTUDIOS CON ENFASIS EN EL ESPA- NOLL EN COSTA RICA}

3.1. Los estudios sobre el español en Costa Rica se remontan a fines del siglo pasado (Gagini 1892, Fernández Ferraz 1892) y principios de este (Quirós 1901), pero haciendo relativa excepción de Agüero (1962, 1964, 1973), Arroyo (1971) y Gaínza (1976), no hay una visión, rigurosa y de conjunto, de los principales fenómenos lingüísticos del castellano en nuestro país. Los trabajos se refieren fundamentalmente al componente léxico (Gagini 1892, 1918, Salesiano 1938, Villegas 1953, 1955, Zúñiga 1976, Garro 1978), y uno solo versa sobre fonología (Chaverría Aguilar 1951). Ninguno de los estudios anteriores tiene como propósito la investigación geográfico-lingüística del español en Costa Rica. De la década de los setenta en adelante, se han publicado investigaciones sobre lé- 
xico (Quesada 1985), fonología (Wilson 1970, Sánchez 1984b), morfología (Vargas Dengo 1974, Constenla 1982), sintaxis (Ross 1982), que muestran, en general, una mayor sustentación teóricolingüística. Recientemente se han escrito tesis sobre la base del paradigma sociolingüísticocuantitativo (Umaña 1981, Quesada Pacheco 1984, Solano 1985). En todo caso, como la región central del país es la de mayor densidad demográfica (aqui se concentra el 57 \% de la población total, según censo de 1984), el habla de esta zona es la que más se ha estudiado. Por otra parte, como la presente investigación llega hasta la época de Agüero, cuya característica metodológica está marcada por el predominio de la escuela lingüística española, no comentamos las obras que se publican después de esa época -esto se incluirá en una investigación de mayor alcance- cuya característica fundamental es el estar enmarcadas en otros modelos lingüísticos (estructural, glosemático, generativo, sociolingüístico-cuantitativo).

\subsection{Estudios globales o incipientes •}

3.2.1. La primera investigación de importancia es el Diccionario de barbarismos y provincialismos de Costa Rica (Gagini 1892), cuyo propósito fundamental era "...el de proporcionar un consultor claro y sencillo a los extranjeros y principalmente a las personas que, deseando expresarse con alguna corrección, no disponen del tiempo necesario para consagrarse a estudios serios de gramática, incluyo también en el presente Diccionario varios artículos relativos a cuestiones puramente gramaticales que ofrecen dificultad en la práctica, como las voces de varia acentuación, el empleo de los derivados verbales, algunos preceptos sobre concordancia y régimen..." (Gagini 1892: IV). El mismo autor (Gagini 1907:5) anota que el castellano en Costa Rica difiere, por causas de sustrato indígena, contacto con lenguas extranjeras, origen peninsular de los colonos y particularidades del ambiente, respecto del castellano en Chile. En la obra Diccionario de costarriqueñismos (Gagini 1918) encontramos el primer paso riguroso en el estudio e individualización del español de Costa Rica respecto de la lengua estándar común o de otras hablas americanas (particularidades de pronunciación, sufijos más usados, etimologías de voces vernáculas); algunas veces ofrece indicaciones de la distribución geográfica general de la palabra o de alguna de sus acepciones:
Quelite. -Quelites o quilites son los brotes o puntas comestibles de la chayotera y ayotera, con las cuales se prepara una especie de cocido. Los indios de Talamanca dan ese nombre a un plato indígena compuesto de varias legumbres.

Quelite en voz náhuatl, quilite, que el P. Rincón traduce por legumbres. En Méjico hay dos plantas que llevan este nombre: el quelite de espiga (Amaranthus hipocondriacus) y el quelite de trigo (Chenophodium viridae).

En Guanacaste es un arbusto de hojas comestibles, pero que ignoramos a cuál especie corresponde (Gagini 1918: 185).

Nanga. -Guan. Barro podrido; en Honduras "estero de fondo pantanoso". En el interior de Costa Rica sólo se usa en la frase "echar ñanga o la ñanga", por morder" (Gagini 1918: 166).

Yo he escuchado "darle un ñangazo a alguien" en el sentido de 'morder'. Por otra parte, Gagini señala que el español de Guanacaste se parece más al de Nicaragua que al del resto de Costa Rica.

Guanacaste. -Geogr. Provincia de Costa Rica, que se extiende desde el Golfo de Nicoya hasta la frontera de Nicaragua. Las costumbres y habla de sus habitantes tienen más parecido con las de este último país que con las del nuestro. Los indígenas de esta región eran aztecas y chia panecas; pero había también tribus caribes (Gagini 1918: 133).

3.2.2. Fernández Ferraz (1892) nos ofrece un estudio etimológico y léxico-gráfico de voces mejicanas de uso corriente en el español de Costa Rica. La obra tiene fundamentalmente un valor historiográfico, ya que el autor desconocía, como la mayoría de americanistas de ese entonces, una metodología científicamente válida para la identificación y análisis etimológicos.

3.2.3. Yoyo Quirós (1901), en un breve articulito, señala algunas palabras y terminachos de uso cotidiano en el español de Costa Rica, con la intención de que los académicos, si así lo deciden, incluyan algunos de esos vocablos en el diccionario: camanance, concertada, china, chacalines, motete, chicas, etc., o para contrastar, preceptivamente, esas formas dialectales respecto de la lengua culta.

3.2.4. EI primer investigador que nos ofrece una descripción de conjunto y bien organizada del español en Costa Rica es D. Arturo Agüero (1962, 1964 y 1973). Desde la perspectiva general del español de América y también sobre la base de diferencias de habla entre dos regiones del país históricamente distintas: el Valle Central y el Guanacaste. Agüero enumera, contrastándolas con la norma 
culta, las principales características fonéticas, gramaticales y léxicas del español en Costa Rica:

Hay matices fonéticos en el español del Valle Central que nos distinguen, no solamente de los hispanohablantes de otros países, sino de los guanacastecos y de los puntarenenses. En la articulación de algunos sonidos, y sobre todo, en la combinación de los elementos prosódicos, las características son notables. Pero las hay generales de América, como el seseo y el yeísmo, y otras, que sin serlo tanto, se registran en ciertas áreas del Continente $-y$ aun de España-, como la pronunciación del grupo tr y residuos arcaicos alojados en zonas rurales a donde no ha llegado o en donde no ha influido mucho la acción docente (Agüero 1962: 126).

El mismo procedimiento emplea al estudiar los otros niveles de la lengua que el autor intitula y organiza en morfología y sintaxis, léxico y semántica. En todo caso, la obra evidencia un interés normativo frente a criterios dialectológicos, tal como lo anotamos anteriormente.

\subsubsection{Víctor Manuel Arroyo (1971), con su obra} El habla popular en la literatura costarricense, ofrece una valiosa aportación al conocimiento del español de Costa Rica. La observación y el estudio del habla popular costarricense que nos proporciona Arroyo, es una investigación rigurosa de la lengua española en nuestro país, cuya limitación estriba en el hecho de que el autnr trabaja únicamente con textos literarios. Arroyo, al presentarnos las características fonéticas, morfosintácticas, léxicas y semánticas del habla costarricense, lo hace comparativamente $y$ en relación con el habla de otras regiones de América y de Europa.

\subsubsection{Gastón Gaínza (1976), en un breve estudio} del español en Costa Rica, reafirma observaciones ya conocidas en trabajos precedentes (Agüero 1962, 1964, 1973); se propone presentar características dialectales que posee la lengua en los núcleos urbanos de la región central del país, con ejemplos ilustrativos de rasgos léxico-semánticos y gramaticales. Con cierto detenimiento el autor se refiere a un problema fonológico -fricación de erre"cuya repercusión puede significar una transformación profunda en el subsistema consonántico de la lengua regional" (Gaínza 1976: 80). Hemos incluido este trabajo en la época de Agüero porque, en cuanto a los presupuestos teóricos, no se aparta en gran medida de los trabajos precedentes, aunque sí representa un segundo momento de esa época, en el entendido de que se les da mayor importancia a los factores sociales como condicionantes de la conducta lingüística de los hablantes.

\subsection{Estudios sobre el componente léxico}

3.3.1. D. Carlos Gagini (1918) es el lexicógrafo costarricense por excelencia. Su obra no ha sido igualada ni en lo relativo a la dimensión ni a la rigurosidad. Cada vez se siente más imperiosa la necesidad de un diccionario de uso del español en Costa Rica, que venga a llenar ese gran vacío dejado por la obra de Gagini, puesto que no ha habido una labor de revisión y actualización de ese diccionario. Este es uno de nuestros propósitos y en él trabajamos.

3.3.2. Salesiano (1938) recoge palabras (diccionario relativamente extenso) y expresiones (doscientos ochenta y dos refranes) que el autor llama Vocabulario Tico, cuyo uso justifica el acopio, que, por su etimología según opinión del autor, entrarían a formar parte del léxico castellano. Aplicado un criterio preceptivo, quizá ni el cincuenta por cien de ese diccionario se acuñe normativamente, ya que "habrá que desechar el cincuenta por ciento (sic) o más, por incorrectas, impropias o mal formadas" (Salesiano 1938: 4). La obra carece de rigurosidad metodológica, fundamentalmente es el trabajo de un buen aficionado, pero sí tiene interés histórico y constituye un rico material para la investigación actual.

3.3.3. Villegas $(1953,1955)$ ofrece una copilación de vocablos y frases del español popular costarricense, cuyas fuentes fueron cuatro novelas nacionales de los años cuarenta, cuatro informantes costarricenses, además de su propia aportación. Ese léxico, que asciende a 1246 palabras, corresponde a lo que el autor llama argot costarricense, en el sentido de que es "el lenguaje que sin ser soez y sin pertenecer a una sola clase de personas, se ha introducido en la lengua diaria de todas las clases sociales" (Villegas 1953: 28), empleándose en ocasiones de familiaridad o camaradería.

\subsubsection{El anglicismo en el habla costarricense (Zú-} ñiga 1976) constituye una reelaboración y selección de su tesis doctoral; se publica veinte años después "ampliado y remozado" (Zúñiga 1976: 16). Es un inventario amplio de anglicismos que muestra la erudición de la autora. Si bien la investigadora dice que su estudio se basa en "el lenguaje oral de nuestro pueblo" (Zúñiga 1976: 15), al no emplearse con rigurosidad criterios de frecuencia, productividad, riqueza semántica, distribución geográfica (Sala y otros: 1977), la investigación toma- 
da en su conjunto produce la impresión de que el español en Costa Rica es anglicado por excelencia, lo cual es falso. No se puede aceptar que aplicación 'solicitud' < inglés application sea de uso general y en todo el país. Se encontrará, sin duda, en un mal traductor. Tampoco puede incluirse en el mismo status lexicográfico un nombre común y un nombre propio, como sucede con Indor Club (Zúñiga 1976: 96). También debe revisarse el criterio diafásico de clasificación del vocablo.

\subsection{5. ¿Habla que el tiempo se lleva? (Garro} 1978) presenta un inventario de 542 palabras que "a juicio del autor se hallan olvidadas en el habla costarricense o están a punto de perderse por el poco empleo que se les da" (Garro 1978: 11-12). Es el trabajo de un buen lector que se interesa por los aspectos normativos de la lengua, particularmente por el léxico, sin formación en nuestra especialidad: desconocimiento de los principios de estructuración de una lengua y de su variación diafásica, diastrática y diatópica. No obstante, es un material útil para ulteriores investigaciones.

\subsection{Estudio sobre fonología}

3.4.1. En el período en comentario, solamente se publica una.investigación sobre el componente fonológico. Oscar Chavarría (1951) en su artículo "The phonemes of Costa Rican Spanish" describe el sistema fonológico del español de Costa Rica, dialecto urbano del Valle Central que comprende las ciudades de San José, Cartago, Alajuela y Heredia. Es un estudio estructural y comparativo respecto del dialecto castellano, tal como lo presentan Tomás Navarro Tomás y George L. Trager. Se aparta, por tanto, de la escuela lingüística española. Como particularidad, el autor postula la existencia de un fonema nasal velar $/ \eta /$ respecto de la alveolar o palatal correspondiente' [Kopéso] / [Kojeso], lo cual vendría a ser una innovación respecto del sistema fonológico castellano.

3.5. Los estudios sobre el español en Costa Rica en el período que hemos llamado de Gagini a Agüero, se caracterizan, en términos generales, por el dominio teórico de la escuela lingüística española (Agüero 1962, 1964, 1973 y Arroyo 1971) o el tratamiento de los dialectalismos como corruptelas de formas peninsulares (Gagini 1982, Quirós 1901, Agüero 1962, 1964, Salesiano 1938, Garro 1978). Cuando hay investigaciones que se apartan de alguna de esas dos constantes o de ambas, se trata de costarricenses que han estudiado en el exterior (Chavarría Aguilar 1951, Villegas 1953, 1955, Zúñiga 1976) o, en el caso de Gaínza, un colega chileno que llega al país después de estudiar en universidades extranjeras. Gagini 1918 sí constituye toda una excepción, quizá a causa de sus contactos con dialectólogos hispanoamericanos, particularmente con R. J. Cuervo.

\section{CONCLUSIONES}

4.1. Los dos tipos de estudios -sobre la lengua castellana o sobre el español en Costa Rica-, comentados anteriormente, tienen, en términos generales, una constante teórica: el considerar la gramática como arte, definida por Nebrija como "scientia recte loquendi recteque scribendi ex doctissimorum virorum usu atque auctoritate collecta" (en Alarcos 1971: 11). El carácter correctivo de esta gramática se manifiesta al promover como norma lingüística la variante culta peninsular (academicistas) o la americana (Bello y sus seguidores) y al proscribir los dialectalismos, puesto que son transgresiones al uso de la gente educada.

4.2. La observación de las 'corruptelas' de una lengua se produce con mayor facilidad en el léxico -actualmente los diccionarios están al alcance de todo aquel que tenga este tipo de preocupaciones-, ya que es el componente de la lengua que, según un sentir general, pide una especialización menor. Como consecuencia de lo anterior, además de la permeabilidad del léxico, tenemos el predominio de trabajos sobre vocabulario -glosarios y diccionarios- y el poco tecnicismo con que se han hecho, en términos generales, las obras lexicográficas costarricenses. Muchos trabajos muestran la erudición de buenos lectores y, al mismo tiempo, un desconocimiento de los principios fundamentales de la lexicográfia.

4.3. Hemos anotado también otra característica general de la época en comentario: el predominio, hasta los setenta, de la escuela lingüística española. Dijimos que si se recurrió a otras corrientes teóricas, los investigadores habían hecho estudios en el exterior. Pues bien, a partir de los setenta -propongamos simbólicamente el año de 1975, cuando se presentan dos tesis de licenciatura en Filología Española sobre la base de dos modelos distintos: neobloomfieldiano (Constenla 1975) o glosemático (Sánchez 1975)- se van a producir cambios de paradigmas en las investigaciones lingüísticas en Cos- 
ta Rica, hecho que se fomentará con la institucionalización de la Sección de Lingüística (hoy Departamento) en la Escuela de Filología, por parte del Dr. Jack Wilson. El desarrollo de esta etapa -cambios de paradigmas lingüísticos- rebasa los objetivos de la presente visión retrospectiva, pero constituirá objeto de estudio de mis investigaciones futuras.

\section{BIBLIOGRAFIA}

Agüero, A. 1962. El español de América y Costa Rica. San José: Lehmann.

1964. "El español de Costa Rica y su atlas lingüístico" en Presente y Futuro de la lengua española. Vol. II. Madrid: Ediciones Cultura Hispánica.

. 1973. El español de América. San José: Editorial Taller.

Alarcos Llorach, E. 1971. Gramática estructural (según la escuela de Copenhague y con especial atención a la lengua española). Madrid: Gredos.

Arroyo, V. 1971. El habla popular en la literatura costarricense. Ciudad Universitaria Rodrigo Facio: Publicaciones de la Universidad de Costa Rica.

Bello, A. 1970 Gramática de la lengua castellana, $8^{a}$ edición, Buenos Aires: Editorial Sopena Argentina.

Brenes Mesén, R. 1905. Gramática histórica y lógica de la lengua castellana. San José: Librería Lehmann.

Constenla Umaña, A. 1975. La lengua guatusa: fonología, gramática y léxico. Tesis de Licenciatura, inédita. Universidad de Costa Rica.

. 1982. "Los Hipocorísticos Costarricenses". Estudios de Lingüística Hispánica. Serie B, Tomo I. San José: Departamento de Lingü ística, Universidad de Costa Rica.

Fernández Ferraz, J. 1892. Nahuatlismos de Costa Rica. Ensayo lexicográfico acerca de las voces mejicanas que se hallan en el habla corriente de los costarricenses. San José: Tipografía Nacional.
Gagini, C. 1892. Diccionario de barbarismos y provincialismos de Costa Rica. San José: Tipografía Nacional.

1907. Elementos de gramática castellana. San José: Tipografía Nacional.

. 1918. Diccionario de costarriqueñismos. San José: Tipografía Nacional.

Gaínza, G. 1976. "El español de Costa Rica: breve consideración acerca de su estudio". Revista de Filología y Lingüistica de la Universidad de Costa Rica II, 4: 79-84.

Garro, J. 1978. ¿Habla que el tiempo se lleva? San José: Editorial Costa Rica.

González, J. 1968. Curso fundamental de gramática castellana. San José: Publicaciones de la Universidad de Costa Rica (3era edición, 1980 San José: UNED).

Quesada, N. 1929. Lecciones de gramática castellana. San José: Lehmann.

Quesada Pacheco, J. 1984. La variación de la /s/ en el área metropolitana de San José: análisis cuantitativo. Tesis para optar el grado de Magister Litterarum, inédita. Universidad de Costa Rica.

Quesada Pacheco, M. 1985. Diccionario regional de los distritos de San Gabriel, Monterrey y la Legua de Aserrí. San José: Editorial Ludovico S.A.

Quirós, Y. 1901. "Hablemos castellano". La Revista No. 561 (San José: Costa Rica) 31 de marzo de 1901 (reedición 1973. Bailar con la más fea. San José: Ministerio de Cultura, Juventud y Deportes).

Ross, R. 1982. Investigando la sintaxis del español San José: UNED.

Salesiano. 1938. Vocabulario de palabras, modismos y refranes ticos (vocabulario tico). Cartago: Escuela Tipográfica Salesiana.

Sánchez, V. 1975. Estudio funcional del morfema modo en griego clásico. Tesis de Licenciatura, inédita. Universidad de Costa Rica. 
. 1984b. "Fricación de erre en el español de Costa Rica: un caso de escisión fonológica". Ponencia para el Congreso Homenaje a Rómulo Gallegos y Pedro Henríquez Ureña, David-Panamá. 23-27 de noviembre, 1984.

Solano, Y. 1985. Formas de tratamiento diádico en el ambiente escolar de San Ramón. Tesis para optar el grado de Magister Litterarum, inédita. Universidad de Costa Rica.

Umaña, J. 1981. Variable vibrants in middle-class Costa Rican Spanish. Tesis de maestría, inédita. Georgetown University.

Vargas Dengo, C. 1974. "El uso de los pronombres 'vos y usted' en Costa Rica". Revista de Ciencias Sociales 8: 7-30.
Villegas, Fr. 1953. El argot costarricense. Tesis doctoral. University of Michigan.

1955. "El argot costarricense". Hispania $38,1: 27-30$.

Wilson, J. 1970. A generative phonological study of Costa Rican Spanish. Tesis doctoral. Michigan University (versión española 1980, "El español de Costa Rica, estudio fonológico generativo". Revista de Filología y Lingüística de la Universidad de Costa Rica VI, 1-2: 3-46).

Zamora, H. 1941. Gramática castellana. San José: Soley y Valverde.

Zúñiga, V. 1976. El anglicismo en el habla costarricense. San José: Editorial Costa Rica. 